\title{
Hyperglycemia Inhibits Recovery From Disuse-Induced Skeletal Muscle Atrophy in Rats
}

\author{
H. KATAOKA ${ }^{1,2}$, J. NAKANO ${ }^{3}$, Y. MORIMOTO ${ }^{1,4}$, Y. HONDA $^{1,4}$, J. SAKAMOTO $^{3}$, \\ T. ORIGUCHI ${ }^{1}$, M. OKITA ${ }^{1}$, T. YOSHIMURA ${ }^{1}$
}

${ }^{1}$ Department of Locomotive Rehabilitation Science, Unit of Rehabilitation Sciences, Nagasaki University Graduate School of Biomedical Sciences, Japan, ${ }^{2}$ Department of Rehabilitation, Nagasaki Memorial Hospital, Japan, ${ }^{3}$ Unit of Physical Therapy and Occupational Therapy Sciences, Nagasaki University Graduate School of Biomedical Sciences, Japan, ${ }^{4}$ Department of Rehabilitation, Nagasaki University Hospital, Japan

Received October 25, 2013

Accepted February 17, 2014

On-line April 3, 2014

\section{Summary}

The purpose of this study was to evaluate the effects of hyperglycemia on skeletal muscle recovery following disuseinduced muscle atrophy in rats. Wistar rats were grouped as streptozotocin-induced diabetic rats and non-diabetic rats. Both ankle joints of each rat were immobilized to induce atrophy of the gastrocnemius muscles. After two weeks of immobilization and an additional two weeks of recovery, tail blood and gastrocnemius muscles were isolated. Serial cross sections of muscles were stained for myosin ATPase $(\mathrm{pH} 4.5)$ and alkaline phosphatase activity. Serum insulin and muscle insulin-like growth factor-1 (IGF-1) levels were also measured. Serum insulin levels were significantly reduced in the diabetic rats compared to the non-diabetic controls. The diameters of type I, IIa, and IIb myofibers and capillary-to-myofiber ratio in the isolated muscle tissue were decreased after immobilization in both treatments. During the recovery period, these parameters were restored in the non-diabetic rats, but not in the diabetic rats. In addition, muscle IGF-1 levels after recovery increased significantly in the non-diabetic rats, but not in the diabetic rats. We conclude that decreased levels of insulin and IGF-1 and impairment of angiogenesis associated with diabetes might be partly responsible for the inhibition of regrowth in diabetic muscle.

\section{Key words}

Hyperglycemia • Disuse-induced muscle atrophy • Recovery

\section{Corresponding author}

J. Nakano, Unit of Physical Therapy and Occupational Therapy Sciences, Nagasaki University Graduate School of Biomedical Sciences, 1-7-1 Sakamoto, Nagasaki-shi 852-8520, Japan. Fax: +81-95-819-7919. E-mail: nakano-j@nagasaki-u.ac.jp

\section{Introduction}

Diabetes mellitus (DM) is often associated with comorbidities such as macrovascular disease, neuropathy, and nephropathy (Brownlee 2005). In addition, skeletal muscle diseases, many of which lead to decreased muscle weight and muscle weakness, have also been observed in diabetes patients and animal models (Krause et al. 2011). Further, skeletal muscle atrophy and hyperglycemia-induced capillary changes in patients with DM have been linked to changes in the expression levels of several hormones and cytokines. For example, the levels of insulin and insulin-like growth factor-1 (IGF-1) were found to be significantly lower in the plasma and skeletal muscle of diabetic animals than that of non-diabetic animals (Fedele et al. 2000, Dehoux et al. 2004). IGF-1 is known to induce skeletal muscle maturation and hypertrophy in an autocrine/paracrine fashion by directly stimulating muscle protein synthesis and/or attenuating protein breakdown (Adams et al. 1999, Glass 2010). The role of IGF-1 as a critical regulator of skeletal muscle growth indicates that reduction in IGF-1 levels may be involved 
in both atrophy and growth inhibition of skeletal muscle in DM patients (Dehoux et al. 2004, Krause et al. 2009). Furthermore, it has been demonstrated that vascular endothelial growth factor-A (VEGF-A) levels are reduced in streptozotocin (STZ)-induced DM, and this reduced expression seems to be a key factor in the associated skeletal muscle capillary changes (Rivard et al. 1999, Kivelä et al. 2006).

Skeletal muscle changes, such as muscle weight loss and myofiber atrophy, are also induced during periods of decreased neuromuscular activity (e.g. gravitational unloading during bed rest, space flights, reflex atrophy, or joint immobilization) (Asmussen et al. 1991, Caiozzo et al. 1997, Zacharová et al. 1997, Kraemer et al. 2000). Importantly, disuse-induced muscle atrophy is reversible and atrophied muscle can regrow upon resumption of regular weight-bearing activity (Nakano et al. 2009, Stevens-Lapsley et al. 2010). Various biological processes, including angiogenesis and growth factor-regulated mechanisms, contribute to skeletal muscle recovery after disuse-induced muscle atrophy. It has been reported that expression of IGF-1 in skeletal muscles is significantly upregulated during muscle regrowth after a period of unloading and cast immobilization, indicating that activation of the IGF-1 signaling pathway is necessary for recovery from disuseinduced muscle atrophy (Nakano et al. 2009, StevensLapsley et al. 2010, Washington et al. 2011). In addition, previous studies also indicate that angiogenesis is strongly correlated with muscle hypertrophy (Plyley et al. 1998, Nakano et al. 2009).

Previously, it has been demonstrated that DM provides an unfavorable environment for the regeneration of skeletal muscles (Vignaud et al. 2007, Jerković et al. 2009, Nguyen et al. 2011). For example, Jerković et al. (2009) reported that the extensor digitorum longus (EDL) myofibers in diabetic rats showed greater atrophy during recovery from muscle injury than did non-diabetic controls. In animal models of ischemia, diabetes has also been shown to impair angiogenesis (Rivard et al. 1999, Hazarika et al. 2007, Fallahzadeh et al. 2011). Although our understanding of diabetes and its associated comorbidities is continually expanding, the effects of diabetes on the recovery from disuse-induced muscle atrophy have not yet been evaluated. Thus, the purpose of the present study was to evaluate the effect of DM on the recovery of skeletal muscle after disuse-induced muscle atrophy in a rat model.

\section{Materials and Methods}

Animals

Male Wistar rats (age, 10 weeks) were purchased from Kudo Laboratories (Tosu, Saga, Japan) and all 42 were housed in the Laboratory Animal Center in Nagasaki University. Rats were housed in standard conditions (temperature, $24{ }^{\circ} \mathrm{C}$; humidity, $60 \% \pm 10 \%$; light, 8:00 $\mathrm{AM}$ to 8:00 $\mathrm{PM}$ ) and were provided unrestricted access to tap water and food pellets. Animal care and experimental procedures were performed in accordance with the Guidelines for Animal Experimentation of Nagasaki University and with approval from the Institutional Animal Care and Use Committee (approval number: 0909090785).

Rats $(n=21)$ received a single intravenous injection of the pancreatic $\beta$-cell toxin STZ (Wako, Osaka, Japan) at $60 \mathrm{mg} / \mathrm{kg}$ in saline solution to induce experimental type 1 diabetes (diabetic). All other rats received a sham injection of an equal volume of saline solution (non-diabetic). To confirm the induction of diabetes, a blood glucose levels were determined three days after the injection. Blood samples were acquired from a tail vein, and blood glucose levels (mg/l) were measured using One Touch Basic Blood glucose Monitoring System (Lifescan Canada Ltd, Burnaby, BC, Canada) and One Touch test strips (Lifescan Canada). Rats were characterized as STZ-diabetic when their blood glucose values were greater than $300 \mathrm{mg} / \mathrm{dl}$. Other symptoms of type 1 diabetes, such as polyuria and weight loss, were also observed in the diabetic rats. Blood glucose levels and body weights (BW) of all rats were recorded once per week to monitor the diabetes. Within three days after verification of diabetes induction, diabetic and non-diabetic rats were used for the experiments described below.

The 21 diabetic rats and 21 non-diabetic rats were randomized equally into 3 groups: Sedentary, cast immobilization only (Immobilization), and recovery after cast immobilization (Recovery). Muscle atrophy was induced in the Immobilization and Recovery groups via cast immobilization as described in a previous study (Okita et al. 2009). Briefly, rats were anesthetized with diethyl ether and their ankle joints were fixed in full plantar flexion with plaster casts, with the triceps surae muscle of the calf immobilized in a shortened position. The casts were kept in place for two weeks. After the period of immobilization, casts of the rats in the Recovery group were removed, and the rats were allowed to recover for two weeks under normal conditions. 


\section{Serum and tissue preparation}

Upon completion of the two-week immobilization period, rats in the Immobilization and Sedentary groups were anesthetized with diethyl ether, tail blood was obtained, and sera were separated and stored at $-80{ }^{\circ} \mathrm{C}$. Next, the gastrocnemius medialis (GM) muscles from both legs were extracted. Rats in the Recovery group were allowed to recover for an additional two weeks following the immobilization period and then sampling was performed using the same methods.

\section{Histochemical analysis}

The GM muscles from the right leg of each animal were frozen with isopentane cooled by liquid nitrogen and transverse serial frozen sections $(6 \mu \mathrm{m})$ were collected using a cryostat (CM1950; Leica, Germany). Diameters of each type of myofiber were determined using myofibrillar adenosine triphosphatase (myosin ATPase) staining (Guth et al. 1970). In the present investigation, the acid pre-incubation solution ( $\mathrm{pH} 4.5)$ was used to classify myofibers from the deep layer of the GM muscle into types I, IIa, and IIb (Zacharová et al. 2005, Novák et al. 2010), which are the primary types of myofibers intermingled in this muscle layer (Delp et al. 1996). The diameters of each type of myofiber were determined using ImageJ software (NIH, Frederick, MD). For each type, more than 200 fiber measurements were recorded per animal.

The alkaline phosphatase reaction, employing an indoxyl-tetrazolium method, was used to visualize capillaries in the muscles. Capillary supply was evaluated based on the capillary density (CD) and capillary-tomyofiber ratio $(\mathrm{C}: \mathrm{F})$. In brief, the capillaries and myofibers were counted in 5 unbiased photographs $\left(\times 100\right.$ magnification; $\left.0.58 \mathrm{~mm}^{2}\right)$ covering the entire area of the deep layer of the muscle. For each photograph, the $\mathrm{CD}$ and $\mathrm{C}: \mathrm{F}$ values were expressed as the number of capillaries per unit area $\left(\mathrm{mm}^{2}\right)$ and the number of capillaries per myofiber, respectively.

Enzyme-linked immunosorbent assay for insulin, IGF-1, and $V E G F$

Serum insulin concentrations were determined using an ELISA kit (Mercodia, Uppsala, Sweden). The deep layer of the GM muscles from the left leg of each animal were trimmed and homogenized in $0.01 \mathrm{M}$ phosphate buffer (PBS; pH 7.4). The levels of IGF-1 and VEGF in the muscle homogenates were also measured using an ELISA kit (Quantikine ${ }^{\circledR}$; R\&D Systems,
Minneapolis, MN, USA).

\section{Statistical analysis}

All data are presented as the mean \pm standard error (SE). Differences between data collected at different time points in the same group were assessed using one-way repeated ANOVA followed by Scheffe post-hoc test. A one-way ANOVA was used to compare all variables among the three groups within the same treatment (diabetic or non-diabetic). In addition, unpaired $t$-tests were used for comparisons between diabetic and non-diabetic rats in the same group. $\mathrm{P}$ values of $<0.05$ were considered statistically significant.

\section{Results}

\section{Body weight and blood glucose}

Table 1 shows the observed changes in blood glucose levels $(\mathrm{mg} / \mathrm{dl})$ and BW (g). For all three groups (Sedentary, Immobilization, and Recovery), blood glucose levels were higher in STZ injection-induced diabetic rats than in the non-diabetic controls. Further, these increased levels were maintained throughout the experimental period. No significant differences in blood glucose levels were observed between Sedentary, Immobilized, and Recovery groups within the diabetic or non-diabetic treatment groups.

BW within 10 days of STZ injection was significantly lower in the Sedentary group of diabetic rats than in the same group of non-diabetic rats. After two weeks of cast immobilization (Immobilization and Recovery groups) BW of both diabetic and non-diabetic rats was significantly lower than that of the Sedentary group rats. The Recovery groups also showed favorable regrowth after the release of cast immobilization, regardless of the severity of diabetes.

\section{Serum insulin levels}

For each group of diabetic rats, serum insulin levels (pmol/l) within four weeks were significantly lower than that in the same group of non-diabetic rats (Table 2). Within the non-diabetic treatment group, serum insulin levels were significantly lower in the Immobilization group than in both the Sedentary and Recovery groups, while no difference was found between the Sedentary and Recovery groups. In contrast, no differences in serum insulin levels were observed in any of the diabetic rat groups, regardless of the hindlimb condition (i.e. sedentary, immobilized, or under recovery). 
Table 1. Changes in blood glucose and body weight.

\begin{tabular}{|c|c|c|c|c|c|c|}
\hline & \multicolumn{3}{|c|}{ Non-diabetic rats } & \multicolumn{3}{|c|}{ Diabetic rats } \\
\hline & Sed & Im & Rec & Sed & Im & Rec \\
\hline \multicolumn{7}{|c|}{$\begin{array}{l}\text { Blood glucose } \\
(\mathrm{mg} / \mathrm{dl})\end{array}$} \\
\hline 0 day & $155.9 \pm 5.5$ & $134.0 \pm 3.7$ & $150.4 \pm 8.0$ & $139.9 \pm 5.2$ & $141.4 \pm 6.2$ & $147.3 \pm 5.2$ \\
\hline 3 days & $136.9 \pm 7.4$ & $147.6 \pm 5.2$ & $142.4 \pm 7.4$ & $584.1 \pm 27.1^{\dagger, *}$ & $513.0 \pm 15.6^{\dagger, \sharp}$ & $509.4 \pm 41.5^{\dagger, *}$ \\
\hline 10 days & $138.1 \pm 5.5$ & $131.0 \pm 5.9$ & $134.3 \pm 8.5$ & $578.4 \pm 18.9^{\dagger, \star}$ & $506.7 \pm 20.5^{\dagger, \star}$ & $527.3 \pm 20.4^{\dagger}$ \\
\hline 17 days & $143.3 \pm 6.9$ & $142.6 \pm 8.7$ & $133.6 \pm 6.6$ & $573.9 \pm 30.1^{\dagger, \star}$ & $585.1 \pm 59.3^{\dagger,}$ & $561.6 \pm 13.1^{\dagger,}$ \\
\hline 24 days & & & $128.6 \pm 6.3$ & & & $533.6 \pm 24.3^{\dagger}$ \\
\hline 31 days & & & $148.9 \pm 8.1$ & & & $590.0 \pm 15.1^{\dagger}$ \\
\hline \multicolumn{7}{|c|}{ Body weight } \\
\hline \multicolumn{7}{|c|}{ (g) } \\
\hline 0 day & $373.7 \pm 7.9$ & $376.6 \pm 5.5$ & $374.9 \pm 6.5$ & $376.7 \pm 4.6$ & $373.6 \pm 5.6$ & $380.2 \pm 4.9$ \\
\hline 3 days & $384.4 \pm 8.2$ & $378.3 \pm 5.7$ & $383.5 \pm 6.4$ & $368.2 \pm 6.5$ & $366.3 \pm 6.8$ & $367.1 \pm 4.2$ \\
\hline 10 days & $406.3 \pm 11.2^{\dagger}$ & $368.1 \pm 3.8^{\S}$ & $371.9 \pm 6.6^{\S}$ & $366.6 \pm 5.9^{\dagger, \dagger}$ & $347.3 \pm 7.8^{\dagger, \star \S}$ & $344.5 \pm 5.0^{\dagger, \ddagger, \S}$ \\
\hline 17 days & $414.1 \pm 11.4^{\dagger}$ & $361.5 \pm 5.8^{\dagger, \S}$ & $362.3 \pm 4.5^{\dagger, \S}$ & $362.7 \pm 7.0^{\dagger, \dagger}$ & $327.0 \pm 7.4^{\dagger, \star \S}$ & $322.8 \pm 7.8^{\dagger, \ddagger, \S}$ \\
\hline 24 days & & & $384.3 \pm 7.3^{\complement}$ & & & $331.0 \pm 6.2^{\dagger,}$ \\
\hline 31 days & & & $406.5 \pm 8.9^{\dagger}$ & & & $348.4 \pm 7.7^{\dagger, \boldsymbol{\oplus},+}$ \\
\hline
\end{tabular}

Data are presented as means $\pm \mathrm{SE}$; $\dagger$ significantly different $(\mathrm{P}<0.05)$ from the same group (i.e. Sedentary, Immobilization, or Recovery) of rats with the same treatment (i.e. non-diabetic or diabetic) at day 0 ; 9 significantly different $(P<0.05)$ from the same group of rats with the same treatment at day $17 ;$; significantly different $(P<0.05)$ from the same group of non-diabetic rats at the same day; $\S$ significantly different $(P<0.05)$ from the Sedentary group of rats with the same treatment at the same day.

Table 2. Serum insulin levels for each group of non-diabetic and diabetic rats.

\begin{tabular}{lcccccc}
\hline & \multicolumn{3}{c}{ Non-diabetic rats } & & \multicolumn{2}{c}{ Diabetic rats } \\
\cline { 2 - 7 } & Sed & Im & Rec & Sed & Im & Rec \\
\hline $\begin{array}{l}\text { Serum insulin } \\
(\text { pmol/l) }\end{array}$ & $563.3 \pm 87.3$ & $187.3 \pm 39.2^{\dagger}$ & $530.2 \pm 96.7^{\ddagger}$ & $79.2 \pm 17.4^{\S}$ & $86.9 \pm 12.4$ & $42.4 \pm 3.1^{\S}$ \\
\hline
\end{tabular}

Data are presented as means $\pm \mathrm{SE} ;+$ significantly different $(\mathrm{P}<0.05)$ from the Sedentary group of rats with the same treatment (i.e. non-diabetic or diabetic); ₹ significantly different $(P<0.05)$ from the Immobilization group of rats with the same treatment; $\S$ significantly different $(P<0.05)$ from the same group of non-diabetic rats.

\section{Myofiber diameter}

The effects of immobilization and reambulation on the size of each type of GM muscle myofiber in nondiabetic and diabetic rats are shown in Figure 1. Comparison of the myofiber diameters in the diabetic and non-diabetic rats in the Sedentary group indicates that there is a significant decrease in type IIa and IIb fibers (Fig. 2B and C), but not in type I fibers (Fig. 2A), in the diabetic rats. The diameters of type I, IIa, and IIb fibers in the Immobilization group of both diabetic and nondiabetic rats decreased significantly compared to the corresponding Sedentary group. In the non-diabetic rats, the diameter of type I, IIa, and IIb fibers in the Recovery group was significantly higher than that in the Immobilization group. In contrast, no differences in diameter were observed between the Recovery and Immobilization groups of the diabetic rats.

\section{Capillaries}

Figure 3 shows the effects of immobilization and reambulation on capillary distribution in GM muscles of non-diabetic and diabetic rats. The CD of the GM muscle was not significantly altered in any of the non-diabetic or diabetic rat groups. However, the $\mathrm{C}: \mathrm{F}$ ratio for diabetic 
rats in the Sedentary group was lower than that of the same group of non-diabetic rats, although the difference was not significant (Table 3). Further, the $\mathrm{C}: \mathrm{F}$ ratio for the Immobilization group was lower than that of the Sedentary group for both the diabetic and non-diabetic treatment groups. In the non-diabetic rats, the $\mathrm{C}: \mathrm{F}$ ratio for the Recovery group was significantly higher than that of the Immobilization group. In contrast, the $\mathrm{C}: \mathrm{F}$ ratio for the Recovery group of the diabetic rats was not significantly higher compared to the Immobilization group (Table 3).

\section{Levels of IGF-1 and VEGF}

In the Sedentary group, IGF-1 and VEGF protein levels in the GM muscles from diabetic rats were decreased compared to non-diabetic rats (Fig. 4). The level of IGF-1 in the non-diabetic Immobilization group was lower than the Sedentary group, while no change was observed between these groups for the diabetic rats. Significantly higher IGF-1 levels were detected in the Recovery group compared to the Immobilization group in the non-diabetic rats, but this increase was not observed for the diabetic rats (Fig. 4A). On the other hand, the nondiabetic and diabetic rats showed a similar trend with respect to the VEGF levels, whereby the Immobilization group was lower than the Sedentary group, and the Recovery group was significantly higher than the Immobilization group (Fig. 4B).
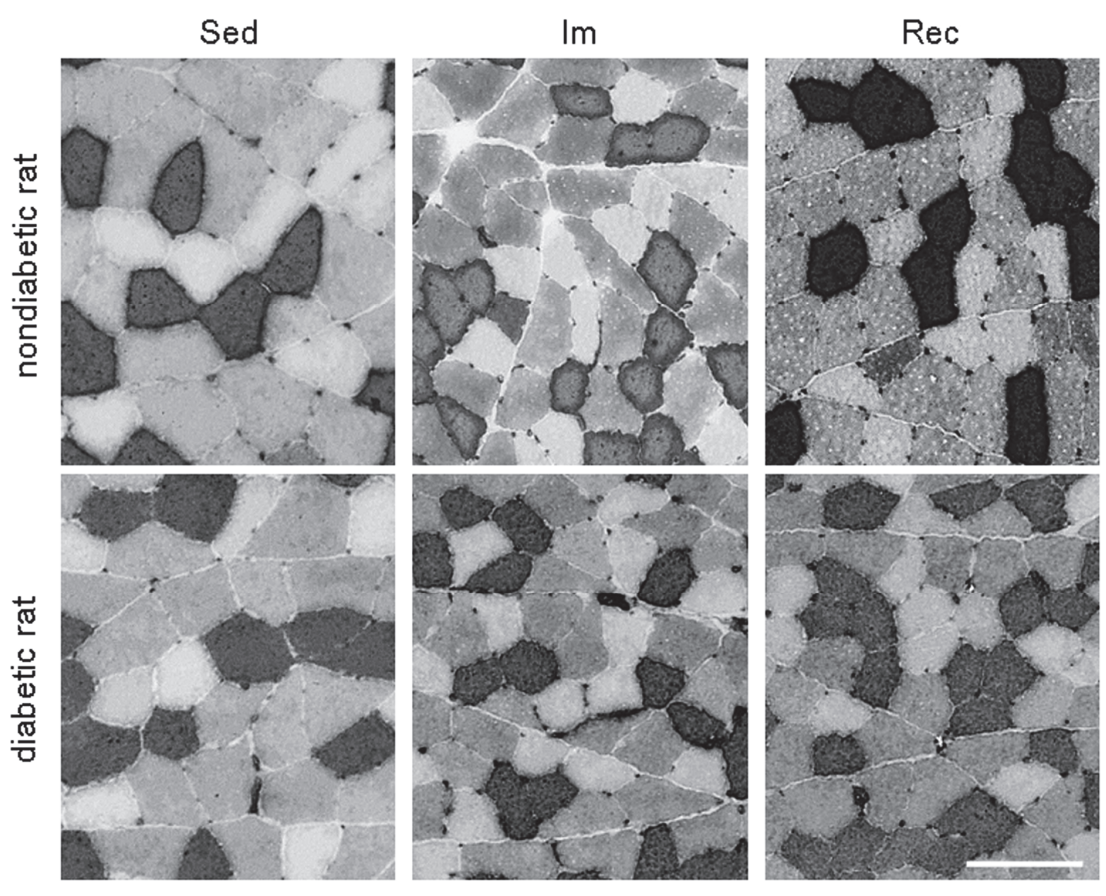

Fig. 1. Light micrographs of histological gastrocnemius muscle sections stained for myosin-ATPase ( $\mathrm{pH}$ 4.5). Type I fibers are visualized as a dark stain; type IIa fibers exhibit a light stain; and type IIb fibers demonstrate an intermediate stain. The upper row represents non-diabetic rats and the lower row represents non-diabetic rats. Scale bar $=50 \mu \mathrm{m}$. Sed - Sedentary; Im Immobilization; Rec - Recovery.
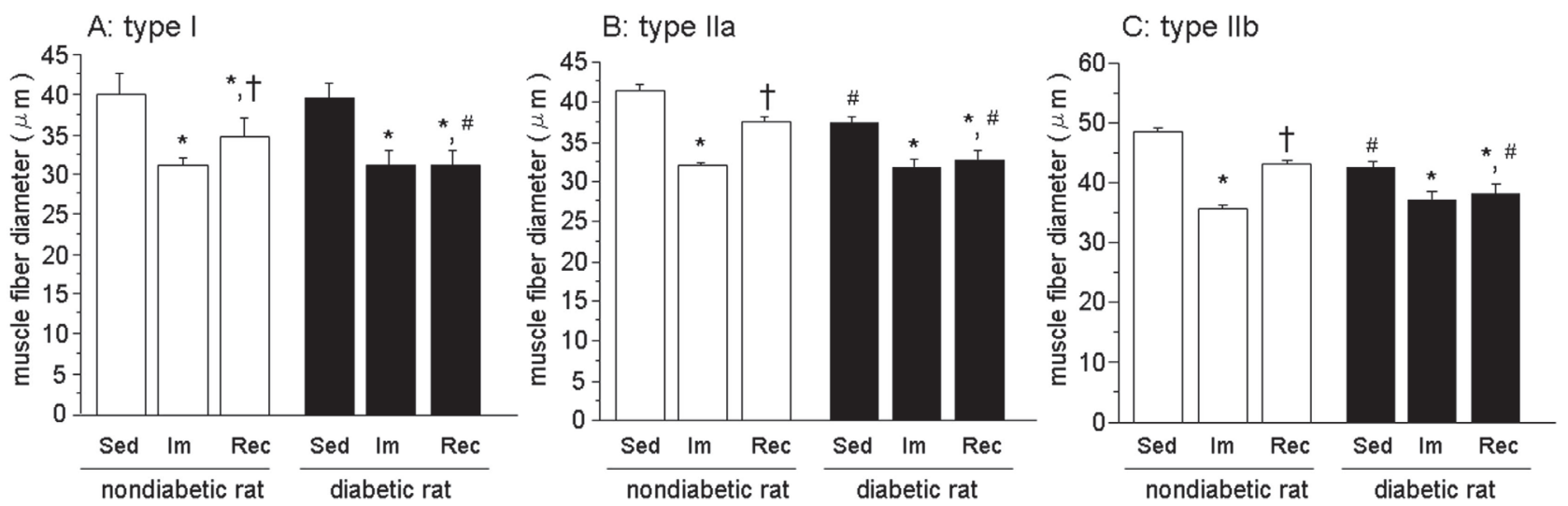

Fig. 2. Diameter of type I (A), IIa (B), and IIb (C) myofibers of gastrocnemius muscle for each group of non-diabetic and diabetic rats. Sed - Sedentary; Im - Immobilization; Rec - Recovery. Data are means $\pm S E ; *$ significantly different $(P<0.05)$ from the Sed group of rats with the same treatment (i.e. non-diabetic or diabetic); + significantly different $(P<0.05)$ from the Im group of rats with the same treatment; \# significantly different $(\mathrm{P}<0.05)$ from the same group of non-diabetic rats. 

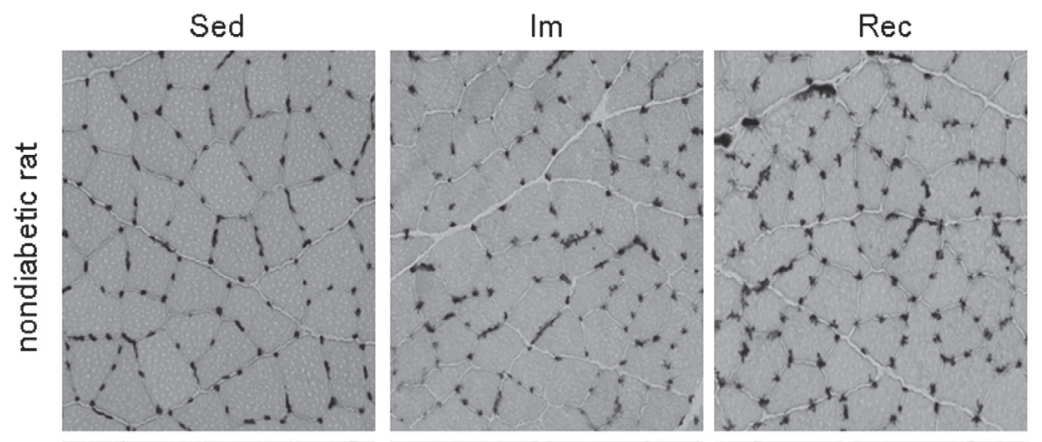

Fig. 3. Light micrographs of histological gastrocnemius muscle sections stained for alkaline phosphatase. Capillaries appear as black dots. The upper row represents non-diabetic rats and the lower row represents non-diabetic rats. Scale bar $=50 \mu \mathrm{m}$. Sed - Sedentary; Im Immobilization; Rec - Recovery.
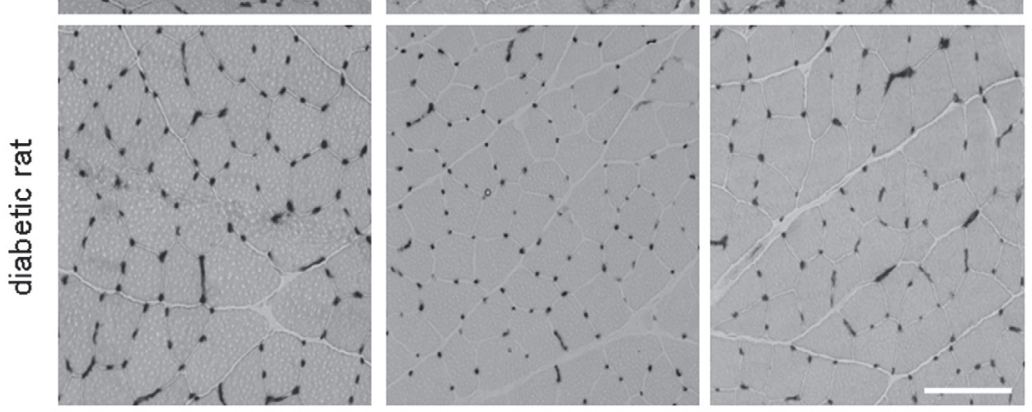

Table 3. Capillary supply in the gastrocnemius muscle in each group of non-diabetic and diabetic rats.

\begin{tabular}{lcccccc}
\hline & \multicolumn{3}{c}{ Non-diabetic rats } & \multicolumn{3}{c}{ Diabetic rats } \\
\cline { 2 - 7 } & Sed & Im & Rec & Sed & Im & Rec \\
\hline $\begin{array}{l}\text { Capillary density } \\
\left(\text { (mm }^{2}\right)\end{array}$ & $800 \pm 30.6$ & $888.9 \pm 30.0$ & $862.6 \pm 38.1$ & $845.9 \pm 44.0$ & $914.9 \pm 63.8$ & $910 \pm 56.9$ \\
$\begin{array}{l}\text { Capillary-to-fiber } \\
\text { ratio }\end{array}$ & $2.79 \pm 0.20$ & $1.91 \pm 0.04^{\dagger}$ & $2.54 \pm 0.07^{\ddagger}$ & $2.4 \pm 0.17$ & $1.70 \pm 0.10^{\dagger}$ & $1.85 \pm 0.07^{\dagger, \S}$ \\
\hline
\end{tabular}

Data are presented as means $\pm \mathrm{SE} ;+$ significantly different $(\mathrm{P}<0.05)$ from the Sedentary group of rats with the same treatment (i.e. non-diabetic or diabetic); ₹ significantly different $(P<0.05)$ from the Immobilization group of rats with the same treatment; $\S$ significantly different $(P<0.05)$ from the same group of non-diabetic rats.
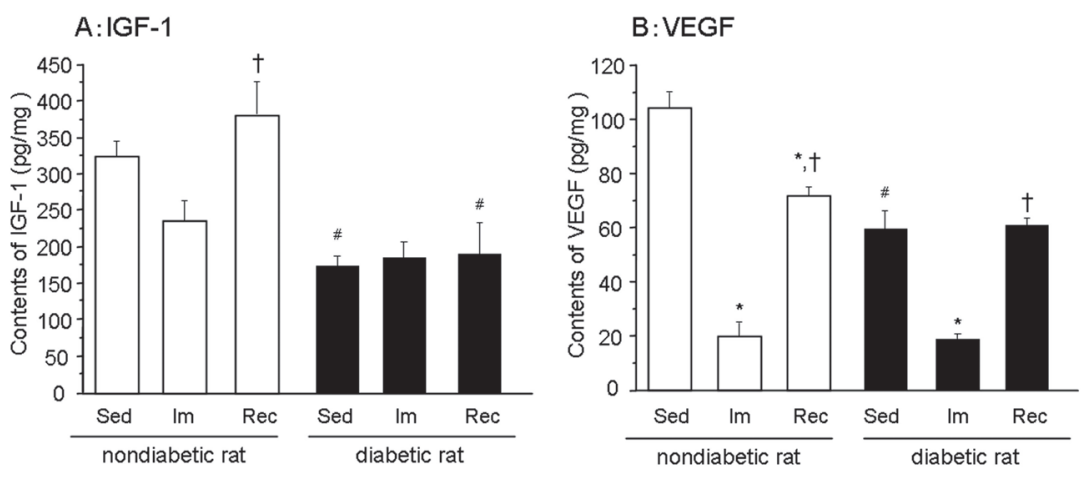

Fig. 4. Levels of IGF-1 (A) and VEGF (B) for each group of non-diabetic and diabetic rats. Sed - Sedentary; Im - Immobilization; Rec - Recovery. Data are means $\pm \mathrm{SE}$; * significantly different $(\mathrm{P}<0.05)$ from the Sed group of rats with the same treatment (i.e. non-diabetic or diabetic); † significantly different $(P<0.05)$ from the Im group of rats with the same treatment; \# significantly different $(P<0.05)$ from the same group of non-diabetic rats.

\section{Discussion}

In this study, we evaluated the effects of hyperglycemia on the recovery of atrophied skeletal muscle myofibers after cast immobilization using an STZ-induced diabetic rat model. Rats are considered severely diabetic when their serum insulin drops below
$80 \mathrm{pmol} / 1$ during a 5 hour fast (Fedele et al. 2000). The mean serum insulin level observed for the Sedentary group of diabetic rats used in this study was $79.2 \mathrm{pmol} / \mathrm{l}$, indicating a severe STZ injection-induced diabetic state. In a previous study, STZ-induced diabetes was shown to cause type II fiber atrophy after the onset of diabetes (Medina-Sanchez et al. 1991). Consistent with this 
finding, the degrees of type I, IIa, and IIb fiber atrophy observed for the Sedentary group of diabetic rats were $2.7 \%, 9.0 \%$, and $11.2 \%$, respectively, relative to the Sedentary group of non-diabetic rats. Moreover, while reduction of the $\mathrm{C}: \mathrm{F}$ ratio in the diabetic mice was not statistically significant, the expression levels of IGF-1 and VEGF were both significantly decreased in the diabetic Sedentary group compared to the non-diabetic controls. These results corroborate data obtained in previous studies (Dehoux et al. 2004, Kivelä et al. 2006, Krause et al. 2011).

In non-diabetic rats, while the blood glucose levels did not differ between groups, the serum insulin levels and $\mathrm{BW}$ in the Immobilization group were significantly lower than that observed in the other groups. These results are similar those of a previous study performed using another rodent model of disuse skeletal muscle atrophy known as hindlimb suspension (Chowdhury et al. 2011). The decrease in BW was also observed in the Immobilization group of diabetic rats; however, the serum insulin levels remained unchanged. It is possible that this lack of change in serum insulin levels was simply because the levels were too low in the diabetic rats to be detected with these methods. With respect to the isolated GM muscle, the Immobilization group of the non-diabetic and diabetic rats showed similar results, whereby myofiber atrophy, reduction of the $\mathrm{C}: \mathrm{F}$ ratio, and reduction of VEGF expression were observed for both treatment groups. Further, the IGF-1 levels were not changed by limb immobilization for either the diabetic or non-diabetic groups. Thus, we suggest that effects of joint immobilization on skeletal muscle are not altered by hyperglycemia.

The most interesting differences between the diabetic and non-diabetic rats in this study were observed during the Recovery stage. After a two-week reambulation period, the degrees of type I, IIa, and IIb myofiber regrowth in the non-diabetic Recovery group were $14.1 \%, 17.0 \%$, and $20.9 \%$, respectively, relative to the Immobilization group. In contrast, the mean diameter of type I, IIa, and IIb myofibers did not increase in the Recovery group of the diabetic rats compared to the Immobilization group, and the levels of regrowth for each type of fiber were only $1.60 \%, 2.54 \%$, and $1.94 \%$, respectively. These results indicate that disuse myofiber atrophy induced by immobilization was not repaired as readily under diabetic conditions. This finding is consistent with previous studies in which the repair of skeletal muscle after injury was impaired in hyperglycemic environments (Vignaud et al. 2007, Jerković et al. 2009, Nguyen et al. 2011).

Current literature indicates that local (paracrine/autocrine) IGF-1 expression plays an important role in skeletal muscle hypertrophy (Krause et al. 2011). IGF-1 signaling through the Akt/mTOR pathway is also a well-described regulator of muscle weight (Glass 2010) and it is known that IGF-1 expression is induced during skeletal muscle regrowth following disuse atrophy in normal animals (Nakano et al. 2009, Stevens-Lapsley et al. 2010, Washington et al. 2011). Here, we observed that the muscle IGF-1 levels in the Recovery group were significantly increased compared to the Immobilization group of non-diabetic rats, but this change did not occur in the diabetic rats. We suspect that the repression of IGF-1 signaling during reambulation in the diabetic rats might be partly responsible for the inhibited myofiber regrowth.

Furthermore, in this study, angiogenesis occurred by reloading in non-diabetic rats, but not in diabetic rats. This result suggests that hyperglycemia impairs endogenous neovascularization in atrophied skeletal muscle, which is consistent with previous reports indicating attenuation of perfusion recovery and angiogenesis in response to ischemia in diabetic animals (Rivard et al. 1999, Fallahzadeh et al. 2011). Chronic reduction of muscle activity (i.e. disuse) is also known to decrease the $\mathrm{C}: \mathrm{F}$ ratio in muscle tissue, which is indicative of a reduction in the absolute number of capillaries (Wagatsuma 2008, Nakano et al. 2009). When atrophied muscle is recovered via reloading after disuse, angiogenesis occurs in conjunction with regrowth of myofibers (Nakano et al. 2009) and is thought to occur in parallel with hypertrophy in skeletal muscle (Plyley et al. 1998). Therefore, we speculate that impaired angiogenesis, in addition to repressed IGF-1 signaling, is a key factor contributing to the lack of myofiber regrowth during the recovery stage in diabetic rats.

Interestingly, the expression of VEGF, a critical factor involved in angiogenesis (Ferrara 1999), during immobilization and reloading was not altered by hyperglycemia in this study. Further, the increase in muscular VEGF levels in the Recovery group compared to the Immobilization group is inconsistent with the impaired angiogenesis observed for the diabetic rats. This contradiction might be explained by a change in the function or expression pattern of VEGF receptors (VEGFR) in the muscle cells. For example, it has been demonstrated that diabetic mice have attenuated 
perfusion recovery following hindlimb ischemia compared to healthy mice, although VEGF expression increased in both mice after ischemia (Hazarika et al. 2007). In addition, membrane embedded and soluble VEGFR-1, which can negatively modulate angiogenic effects, were significantly increased in diabetic mice in comparison to healthy mice (Hazarika et al. 2007). Furthermore, Rabinovsky et al. (2004) reported that plasmid IGF-1 delivery and sequestration during muscle regeneration after injury could be done to augment angiogenesis and increase blood flow in the diabetic mice, indicating that IGF-1 may play a more important role than VEGF during angiogenesis in muscle. Therefore, it is probable that the suppression of IGF-1 we observed during reloading in the diabetic rats is the primary mechanism associated with the inhibition of angiogenesis, regardless of the stable expression of VEGF in the muscle tissue.

In conclusion, our study demonstrates that hyperglycemia inhibits the recovery from disuse muscle atrophy induced by cast immobilization through prevention of angiogenesis and regrowth of all types of myofiber in the muscle tissue. Hyperglycemia-related muscle recovery inhibition might, at least in part, explain the muscle weakness observed in patients with DM, especially during rehabilitation after casting and bed rest. Although additional research is necessary to elucidate the mechanistic links and potential clinical implications, our data represent a step towards uncovering the relationship between DM and skeletal muscle complications.

\section{Conflict of Interest}

There is no conflict of interest.

\section{Acknowledgements}

We would like to thank Nao Yoshida and Shogo Nishikawa of Nagasaki Memorial Hospital for their help in the data analysis.

\section{References}

ADAMS GR, HADDAD F, BALDWIN KM: Time course of changes in markers of myogenesis in overloaded rat skeletal muscles. J Appl Physiol 87: 1705-1712, 1999.

ASMUSSEN G, SOUKUP T: Arrest of developmental conversion of type II to type I fibres after suspension hypokinesia. Histochem J 23: 312-322, 1991.

BROWNLEE M: The pathobiology of diabetic complications: a unifying mechanism. Diabetes 54: 1615-1625, 2005.

CAIOZZO VJ, BAKER MJ, MCCUE SA, BALDWIN KM: Single-fiber and whole muscle analyses of MHC isoform plasticity: interaction between T3 and unloading. Am J Physiol 273: C944-C952, 1997.

CHOWDHURY P, SOULSBY ME, JAYROE J, AKEL NS, GADDY D, DOBRETSOV M: Pressure hyperalgesia in hind limb suspended rats. Aviat Space Environ Med 82: 988-991, 2011.

DEHOUX M, VAN BENEDEN R, PASKO N, LAUSE P, VERNIERS J, UNDERWOOD L, KETELSLEGERS JM, THISSEN JP: Role of the insulin-like growth factor I decline in the induction of atrogin-1/MAFbx during fasting and diabetes. Endocrinology 145: 4806-4812, 2004.

DELP MD, DUAN C: Composition and size of type I, IIA, IID/X, and IIB fibers and citrate synthase activity of rat muscle. J Appl Physiol 80: 261-270, 1996.

FALLAHZADEH AR, KHAZAEI M, SHARIFI MR: Restoration of angiogenesis by enalapril in diabetic hindlimb ischemic rats. Biomed Pap Med Fac Univ Palacky Olomouc Czech Repub 155: 137-142, 2011.

FEDELE MJ, HERNANDEZ JM, LANG CH, VARY TC, KIMBALL SR, JEFFERSON LS, FARRELL PA: Severe diabetes prohibits elevations in muscle protein synthesis after acute resistance exercise in rats. J Appl Physiol 88: 102-108, 2000.

FERRARA N: Molecular and biological properties of vascular endothelial growth factor. J Mol Med (Berl) 77: 527-543, 1999.

GLASS DJ: PI3 kinase regulation of skeletal muscle hypertrophy and atrophy. Curr Top Microbiol Immunol 346: 267-278, 2010.

GUTH L, SAMAHA FJ: Procedure for the histochemical demonstration of actomyosin ATPase. Exp Neurol 28: 365-367, 1970. 
HAZARIKA S, DOKUN AO, LI Y, POPEL AS, KONTOS CD, ANNEX BH: Impaired angiogenesis after hindlimb ischemia in type 2 diabetes mellitus: differential regulation of vascular endothelial growth factor receptor 1 and soluble vascular endothelial growth factor receptor 1. Circ Res 101: 948-956, 2007.

JERKOVIĆ R, BOSNAR A, JURISIĆ-ERZEN D, AZMAN J, STARCEVIĆ-KLASAN G, PEHAREC S, COKLO M: The effects of long-term experimental diabetes mellitus type I on skeletal muscle regeneration capacity. Coll Antropol 33: 1115-1119, 2009.

KIVELÄ R, SILVENNOINEN M, TOUVRA AM, LEHTI TM, KAINULAINEN H, VIHKO V: Effects of experimental type 1 diabetes and exercise training on angiogenic gene expression and capillarization in skeletal muscle. FASEB J 20: 1570-1572, 2006.

KRAEMER WJ, STARON RS, GORDON SE, VOLEK JS, KOZIRIS LP, DUNCAN ND, NINDL BC, GÓMEZ AL, MARX JO, FRY AC, MURRAY JD: The effects of 10 days of spaceflight on the shuttle Endeavor on predominantly fast-twitch muscles in the rat. Histochem Cell Biol 114: 349-355, 2000.

KRAUSE MP, RIDDELL MC, GORDON CS, IMAM SA, CAFARELLI E, HAWKE TJ: Diabetic myopathy differs between Ins2Akita+/- and streptozotocin-induced Type 1 diabetic models. $J$ Appl Physiol 106: 1650-1659, 2009.

KRAUSE MP, RIDDELL MC, HAWKE TJ: Effects of type 1 diabetes mellitus on skeletal muscle: clinical observations and physiological mechanisms. Pediatr Diabetes 12: 345-364, 2011.

MEDINA-SANCHEZ M, RODRIGUEZ-SANCHEZ C, VEGA-ALVAREZ JA, MENEDEZ-PELAEZ A, PEREZCASAS A: Proximal skeletal muscle alterations in streptozotocin-diabetic rats: a histochemical and morphometric analysis. Am J Anat 191: 48-56, 1991.

NAKANO J, KATAOKA H, SAKAMOTO J, ORIGUCHI T, OKITA M, YOSHIMURA T: Low-level laser irradiation promotes the recovery of atrophied gastrocnemius skeletal muscle in rats. Exp Physiol 94: 1005-1015, 2009.

NGUYEN MH, CHENG M, KOH TJ: Impaired muscle regeneration in ob/ob and db/db mice. ScientificWorldJournal 11: 1525-1535, 2011.

NOVÁK P, ZACHAŘOVÁ G, SOUKUP T: Individual, age and sex differences in fiber type composition of slow and fast muscles of adult Lewis rats: comparison with other rat strains. Physiol Res 59: 783-801, 2010.

OKITA M, NAKANO J, KATAOKA H, SAKAMOTO J, ORIGUCHI T, YOSHIMURA T: Effects of therapeutic ultrasound on joint mobility and collagen fibril arrangement in the endomysium of immobilized rat soleus muscle. Ultrasound Med Biol 35: 237-244, 2009.

PLYLEY MJ, OLMSTEAD BJ, NOBLE EG: Time course of changes in capillarization in hypertrophied rat plantaris muscle. J Appl Physiol 84: 902-907, 1998.

RABINOVSKY ED, DRAGHIA-AKLI R: Insulin-like growth factor I plasmid therapy promotes in vivo angiogenesis. Mol Ther 9: 46-55, 2004.

RIVARD A, SILVER M, CHEN D, KEARNEY M, MAGNER M, ANNEX B, PETERS K, ISNER JM: Rescue of diabetes-related impairment of angiogenesis by intramuscular gene therapy with adeno-VEGF. Am J Pathol 154: 355-363, 1999.

STEVENS-LAPSLEY JE, YE F, LIU M, BORST SE, CONOVER C, YARASHESKI KE, WALTER GA, SWEENEY HL, VANDENBORNE K: Impact of viral-mediated IGF-I gene transfer on skeletal muscle following cast immobilization. Am J Physiol Endocrinol Metab 299: E730-E740, 2010.

VIGNAUD A, RAMOND F, HOURDÉ C, KELLER A, BUTLER-BROWNE G, FERRY A: Diabetes provides an unfavorable environment for muscle mass and function after muscle injury in mice. Pathobiology 74: 291-300, 2007.

WAGATSUMA A: Effect of hindlimb unweighting on expression of hypoxia-inducible factor-1alpha vascular endothelial growth factor, angiopoietin, and their receptors in mouse skeletal muscle. Physiol Res 57: 613-620, 2008.

WASHINGTON TA, WHITE JP, DAVIS JM, WILSON LB, LOWE LL, SATO S, CARSON JA: Skeletal muscle mass recovery from atrophy in IL-6 knockout mice. Acta Physiol (Oxf) 202: 657-669, 2011.

ZACHAROVÁ G, KNOTKOVÁ-URBANCOVÁ H, HNÍK P, SOUKUP T: Nociceptive atrophy of the rat soleus muscle induced by bone fracture: a morphometric study. J Appl Physiol 82: 552-557, 1997. 
ZACHAROVÁ G, VADÁSZOVÁ A, SMERDU V, ASMUSSEN G, SOUKUP T: The effect of a unilateral muscle transplantation on the muscle fiber type and the MyHC isoform content in unoperated hind limb slow and fast muscles of the inbred Lewis rats. Physiol Res 54: 691-696, 2005. 